\title{
A Retrospective Clinical View of Basal Cell Carcinoma and Squamous Cell Carcinoma in the Head and Neck Region: A Single Institution's Experience of 247 Cases over 19 Years
}

Kyung Won Kang, Dong Lark Lee, Hea Kyeong Shin, Gyu Yong Jung, Joon Ho Lee, Myeong Su Jeon

Department of Plastic and Reconstructive Surgery, Dongguk University College of Medicine, Gyeongju, Korea

No potential conflict of interest relevant to this article was reported.

\begin{abstract}
Background: The two most common skin cancers are basal cell carcinoma (BCC) and squamous cell carcinoma (SCC). The purpose of this study was to describe the detailed clinical behavior of BCC and SCC in the head and neck region over 19 years at a single institution.

Methods: A retrospective analysis was performed for all patients with non-melanoma skin cancer who had undergone surgical resection over an 18-year period. Patient charts were reviewed for demographic information, tumor size, onset-to-diagnosis, anatomic location, clinical subtype, histologic differentiation, method of surgical treatment, and recurrence.

Results: The review identified 265 cases of either BCC or SCC in 226 patients. Of the 226 patients, $80(35.4 \%)$ were men and $146(64.6 \%)$ were women. BCC ( $n=138,55.9 \%)$ was more frequent than SCC $(109,44.1 \%)$. The most frequent age group was 70 -to-79 year olds (45 patients, $35.2 \%$ ) for BCC and 80 -to- 89 year olds (41 patients, $41.8 \%$ ) for SCC. By aesthetic units of the face, the most common location was the nasal unit (44 cases, $31.9 \%$ ) for BCC and the buccal unit (23 cases, $21.1 \%$ ) for SCC. The most common clinical subtype of BCC was the nodular type (80 cases, $58.0 \%$ ). Local flaps were most commonly used to cover surgical defects (136 cases, $55.1 \%)$. Recurrent rates were $2.2 \%$ for BCC and $5.5 \%$ for SCC.

Conclusion: In our study, many characteristics of BCC and SCC were compared to previously published reports were generally similar, except the ratio of BCC to SCC. Further study can help to establish the characteristics of BCC and SCC.
\end{abstract}

Keywords: Skin neoplasms / Carcinoma, basal cell / Carcinoma, squamous cell

\section{INTRODUCTION}

Skin cancer is the most common type of cancer and affects over 2 million people annually in the United States [1,2]. Skin cancers are distinguished by the type of cells that give rise to each lesion, and

\footnotetext{
Correspondence: Dong Lark Lee

Department of Plastic and Reconstructive Surgery, Dongguk University College of Medicine, 87 Dongdae-ro, Gyeongju 780-350, Korea

E-mail: drldr@paran.com

*This article was presented as a free paper at the 72nd Congress of the Korean Society of Plastic and Reconstructive Surgeons on November 7-9, 2014 in Seoul, Korea.

Received June 24, 2015 / Revised August 16, 2015 / Accepted December 3, 2015
}

consist of basal cell carcinoma (BCC) (77\%), squamous cell carcinoma (SCC) (20\%), malignant melanoma (3\%), and rare tumors of cutaneous origin $(<1 \%)[3,4]$. Anatomic distribution is heavily skewed towards head and neck area for skin cancers, as $85 \%$ of BCCs and $75 \%$ of SCCs occur in this region [1].

BCC is characterized by slow growth and rare metastasis [1,4], whereas SCC is typically more aggressive with respect to invasion and metastasis [1]. Many factors influence the development of BCC and SCC, with sun exposure being the most prominent factor $[1,4,5]$.

Cutaneous malignant tumors are being reported with increas- 
ing frequency in the Korean population. Currently, the population experiences incidences of $0.25 \%$ and $0.34 \%$ for BCC and SCC, respectively [6]. Among various methods of treating BCC and SCC, wide local excision with frozen section or Mohs surgery is the standard, as each of these options minimizes local recurrence $[1,4,5]$.

This study was undertaken to analyze many characteristics and treatment methods of BCC and SCC in the head and neck region and to compare with previously published reports.

\section{METHODS}

A retrospective review was performed for all patients treated for BCC or squamous carcinoma at our institution between April 1997 and March 2015. Multiple concurrent lesions in a single patient were considered as a single case. Patient charts were reviewed for demographic information, tumor size, onset-to-diagnosis, anatomic location, clinical subtype, histologic differentiation, method of surgical treatment, and recurrence.

All of the tumors were resected with negative margins on intraoperative frozen sections. Tissue defects were closed primarily whenever possible. Otherwise, larger defects were resurfaced using skin graft or local flap.

\section{RESULTS}

The review identified 265 cases of either BCC or SCC in 226 patients. Of the 226 patients, 80 (35.4\%) were men and 146 (64.6\%) were women. BCC $(n=138,55.9 \%)$ was more frequent than SCC $(109,44.1 \%)$. The male-to-female ratio was 1:1.4 (53 vs. 75 patients) for BCC and 1:2.6 (27 vs. 71 patients) for SCC. Patient ages ranged from 28 to 93 years (mean 70.8 years) for BCC and from 54 to 97 years (mean 79.9 years) for SCC. The most frequent age group was 70-to-79 year olds (45 patients, 35.2\%) for BCC and 80-to-89 year olds (41 patients, $41.8 \%$ ) for SCC (Fig. 1).

\section{Tumor size and onset-to-diagnosis}

Tumor diameters were between 1 and $2 \mathrm{~cm}$ in $50 \%$ of BCC cases (69 cases) and in $49.6 \%$ of SCC cases ( 54 cases). The average tumor diameter was $1.15 \mathrm{~cm}$ for BCC and $1.65 \mathrm{~cm}$ for SCC (Fig. 2). For BCC, the onset-to-diagnosis ranged from 0.75 to 20 months with a mean delay of 3.2 months. For SCC, it ranged from 1 to 18 months with a mean delay of 1.9 months. The number of patients treated for BCC and SCC at our institution had increased for each year under the review period (Fig. 3).
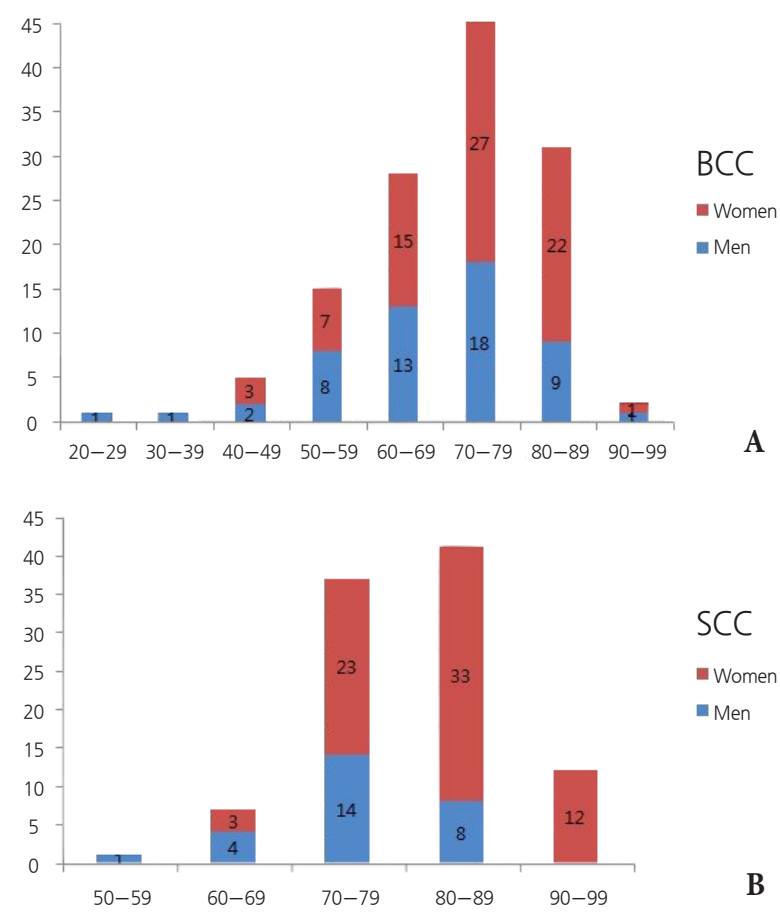

Fig. 1. (A, B) Age distributions of the skin cancer patients by age and sex. BCC, basal cell carcinoma; SCC, squamous cell carcinoma.

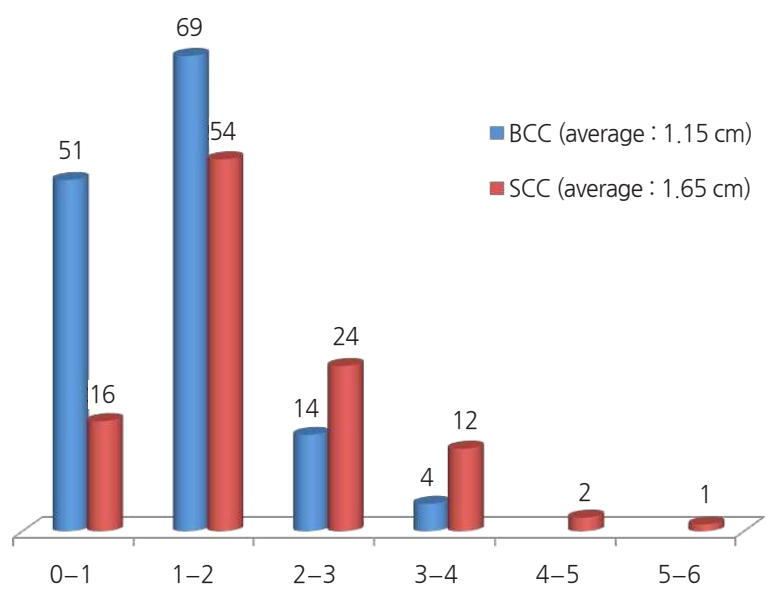

Fig. 2. Lesion diameters. Distribution of maximum lesion diameters for BCC and SCC. BCC, basal cell carcinoma; SCC, squamous cell carcinoma. 


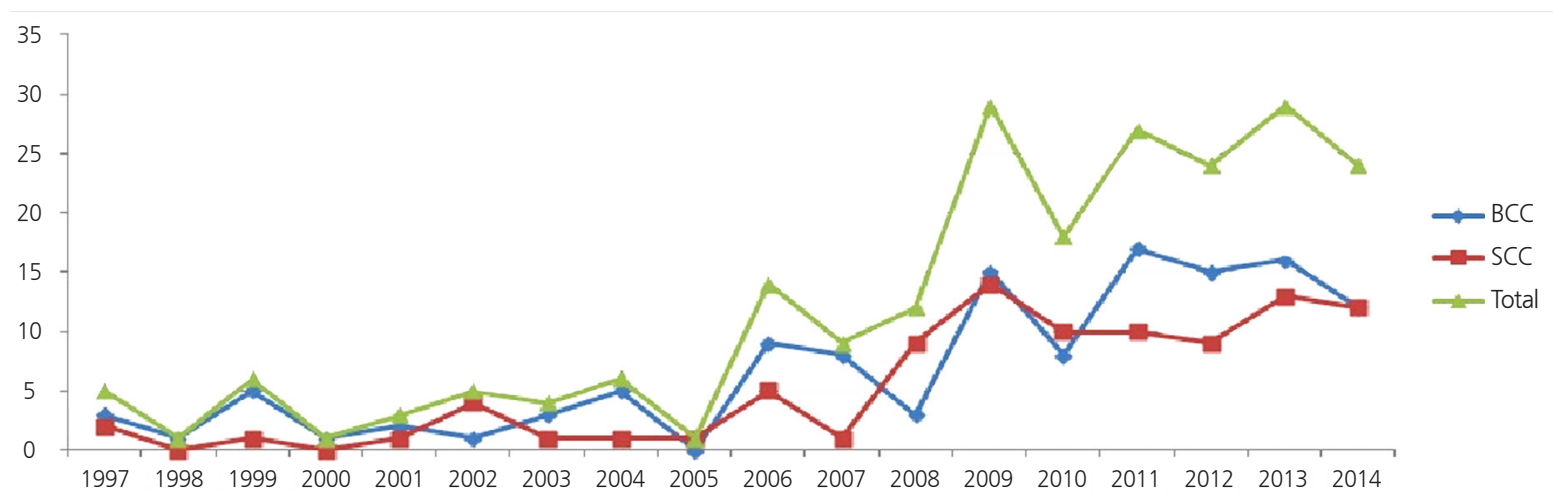

Fig. 3. Numbers of patients. The patients treated for skin cancer at our institute from 1997 to 2014 . BCC, basal cell carcinoma; SCC, squamous cell carcinoma.

\section{Anatomic distribution}

Of the 265 tumors, the vast majority ( $\mathrm{n}=247,93.2 \%)$ was located in the head and neck area. The proportion of tumors in the head and neck region was 93.2\% (138 of 148 cases) for BCC and 93.2\% (109 of 117 cases) for SCC.

The location of head and neck tumors were further analyzed by reviewing the preoperative photographs and categorizing each tumor as belonging to one of the 13 aesthetic units of the face (Fig. 4). BCC showed predilection for the nasal unit (44 cases, 31.9\%), followed by the orbital (31 cases, 22.5\%) and the infraorbital (14 cases, 10.1\%) units. In contrast, squamous cell carcinomas were most commonly in the buccal unit (23 cases, $21.1 \%$ ), followed by the zygomatic (20 cases, $18.3 \%$ ) and the temporal (15 cases, 13.8\%) units (Fig. 5).

\section{Clinical subtype for BCC}

The 138 cases of BCC were divided into four clinical subtypes (nodular, pigmented, superficial, and morpheaform) using preoperative photographs (Fig. 6) [4]. The most common clinical subtype was the nodular type ( 80 cases, $58.0 \%$ ), followed by the pigmented type (38 cases, 27.5\%) (Fig. 7).

\section{Histologic differentiation for SCC}

The histologic reports were reviewed for the 109 cases of SCC, and
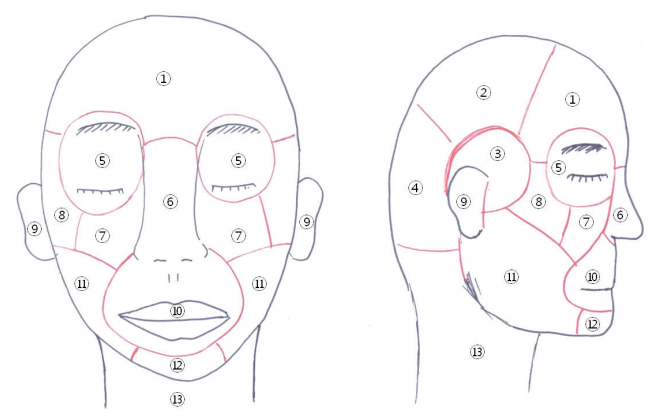

Fig. 4. Aesthetic units of the head and neck area. 1, frontal; 2, parietal; 3 , temporal; 4, occipital; 5, orbital; 6, nasal; 7, infraorbital; 8, zygomatic; 9 , auricular; 10 , oral; 11, buccal; 12 , mental; 13 , neck.

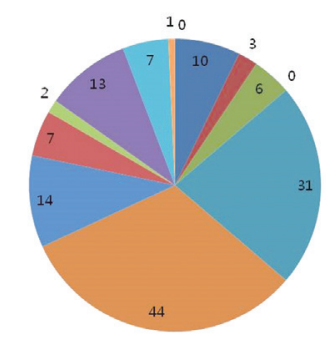

BCC

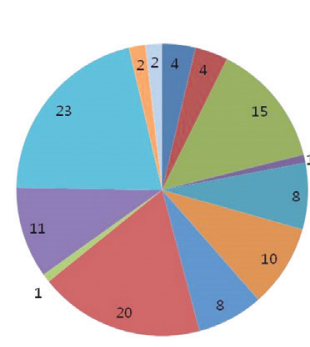

SCC

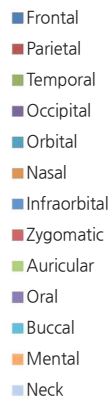

= Neck
Fig. 5. Aesthetic unit. Distributions of BCC and SCC by aesthetic unit in the head and neck area. BCC, basal cell carcinoma; SCC, squamous cell carcinoma.

each SCC tumor was classified as either well, moderately, or poorly differentiated. Of the 109 cases, histologic classification was unavailable for 30 cases. These cases were categorized as "unknown" (Fig. 8). 

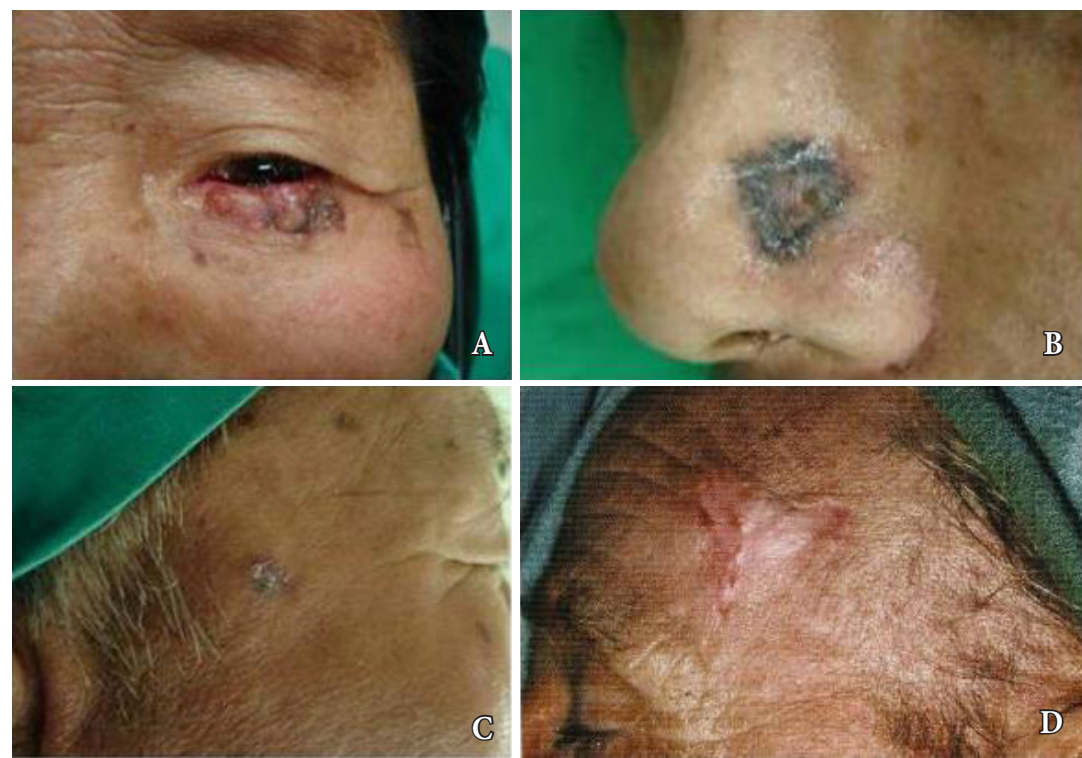

Fig. 6. The four clinical subtypes of basal cell carcinoma. (A) Nodular. (B) Pigmented. (C) Superficial. (D) Morpheaform.

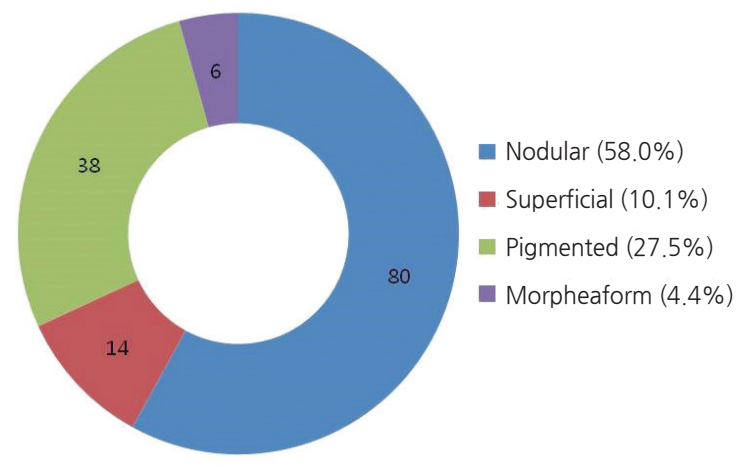

Fig. 7. Distribution of basal cell carcinoma by clinical subtype.

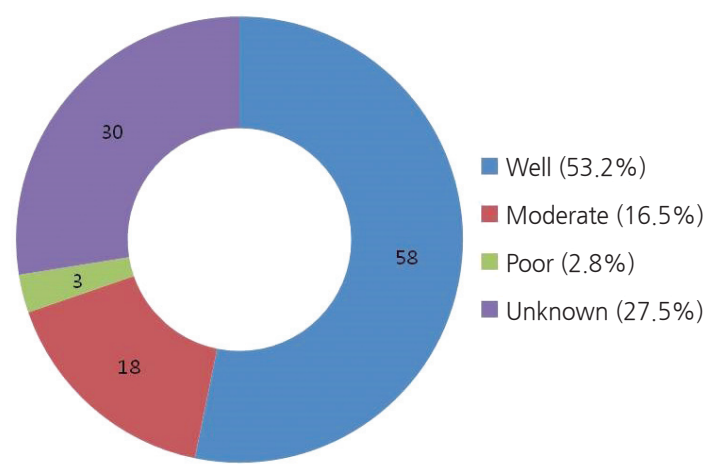

Fig. 8. Distribution of SCC based on histologic differentiation. SCC, squamous cell carcinoma.

\section{Surgical treatment: operative methods}

As per our retrospective inclusion criteria, all of the 247 tumors had been treated with wide excision. The most commonly employed method of wound closure was local flap (136 cases, 55.1\%), followed by primary closure ( 66 cases, $26.7 \%$ ) and FTSG ( 38 cases, 15.4\%). Among local flaps, the most commonly used flap type was V-Y advancement flap (54 cases) followed by a transposition flap (22 cases). The postauricular area (27 cases) was most donor site for a FTSG. Local flap was the most commonly used method for covering surgical defects after wide excision of BCC (79 cases, $57.2 \%$ ) and SCC (57 cases, 52.3\%). Primary repair was the second most commonly used method for BCC (32 cases, 23.2\%) and for SCC (34 cases, 31.2\%).

Operative methods were also analyzed by aesthetic units. A local flap was most commonly used to repair surgical defects in the frontal ( 6 of 14 cases, $42.9 \%$ ), temporal ( 8 of 21 cases, $38.1 \%$ ), occipital (1 of 1 case, 100\%), orbital (21 of 39 cases, 53.8\%), nasal (34 of 54 cases, $63.0 \%$ ), infraorbital (14 of 22 cases, 63.6\%), zygomatic (15 of 27 cases, $55.6 \%$ ), oral ( 15 of 24 cases, $62.5 \%$ ), buccal (18 of 30 cases, $60.0 \%$ ), and neck areas (1 of 2 cases, $50.0 \%$ ). Split-thickness skin grafts were most commonly used to repair defects in the parietal area ( 5 of 7 cases, $71.4 \%)$. Primary repair was used to repair defects 
in the auricular ( 2 of 3 cases, 66.7\%), mental ( 2 of 3 cases, $66.7 \%$ ), and neck area ( 1 of 2 cases) (Table 1 ).

\section{Recurrence}

Of the 247 cases, local recurrence had occurred in 9 cases (3.6\%)

Table 1. Locations and operative methods used to treat BCC and SCC

\begin{tabular}{lccccc}
\multirow{2}{*}{$\begin{array}{l}\text { Aesthetic } \\
\text { unit }\end{array}$} & \multicolumn{5}{c}{ No. of patients (BCC/SCC [total]) } \\
\cline { 2 - 6 } Frontal & $\begin{array}{c}\text { Local } \\
\text { flap }\end{array}$ & FTSG & STSG & $\begin{array}{c}\text { Primary } \\
\text { closure }\end{array}$ & Total \\
\hline Parietal & $0 / 1(6)$ & $2 / 1(3)$ & $1 / 0(1)$ & $2 / 2(4)$ & $10 / 4(14)$ \\
\hline Temporal & $1 / 7(8)$ & 0 & $3 / 2(5)$ & 0 & $3 / 4(7)$ \\
\hline Occipital & $0 / 1(1)$ & 0 & 0 & 0 & $0 / 1(1)$ \\
\hline Orbital & $19 / 2(21)$ & $4 / 1(5)$ & 0 & $8 / 5(13)$ & $31 / 8(39)$ \\
\hline Nasal & $28 / 6(34)$ & $10 / 3(13)$ & 0 & $6 / 1(7)$ & $44 / 10(54)$ \\
\hline Intraorbital & $10 / 4(14)$ & $1 / 3(4)$ & 0 & $3 / 1(4)$ & $14 / 8(22)$ \\
\hline Zygomatic & $5 / 10(15)$ & $1 / 1(2)$ & 0 & $1 / 9(10)$ & $7 / 20(27)$ \\
\hline Auricular & 0 & $0 / 1(1)$ & 0 & $2 / 0(2)$ & $2 / 1(3)$ \\
\hline Oral & $8 / 7(15)$ & $3 / 0(3)$ & 0 & $2 / 4(6)$ & $13 / 11(24)$ \\
\hline Buccal & $3 / 15(18)$ & $0 / 1(1)$ & 0 & $4 / 7(11)$ & $7 / 23(30)$ \\
\hline Mental & $0 / 1(1)$ & 0 & 0 & $1 / 1(2)$ & $1 / 2(3)$ \\
\hline Neck & $0 / 1(1)$ & 0 & 0 & $0 / 1(1)$ & $0 / 2(2)$ \\
\hline Total & $79 / 57(136)$ & $23 / 15(38)$ & $4 / 3(7)$ & $32 / 34(66)$ & $138 / 109(247)$ \\
\hline
\end{tabular}

BCC, basal cell carcinoma; SCC, squamous cell carcinoma; FTSG, full thickness skin graft; STSG, split thickness skin graft. over the 19 year study periods. Of the recurrent tumors, 3 were of BCC and 6 were of SCC with recurrent rates of 2.2\% and 5.5\% for BCC and SCC, respectively. Secondary operation was curative without recurrence in 6 cases with a mean follow-up of 22 months. However, secondary operation was followed by recurrence in one patient at 10 months, who was lost to follow-up. The other two patients did not undergo a secondary operation at our hospital after receiving a diagnosis of recurrence (Table 2).

\section{DISCUSSION}

Skin cancer is the most common type of cancer, with incidence increasing worldwide over the last decade [3,4]. The most common form of skin cancer is BCC, which accounts for $77 \%$ of all skin cancers [2]. The second most common skin cancer is squamous cell carcinoma, at $20 \%$ of all skin cancers [4,6]. Other skin cancers, such as malignant melanoma, are relatively rare.

For the most part, the non-melanoma skin cancers are more frequent in the elderly population, which reflects the relationship between cumulative sun exposure and cancer development $[4,5]$. A number of epidemiologic studies conducted in Korea have revealed such increases in incidence of skin cancer, with the study by Kim et al. demonstrating skin cancer incidence increasing from $0.25 \%$ to $0.34 \%$ between two periods separated by a decade [6].

In the present study, BCC patients were 28 to 93 years of age,

Table 2. Review of the 9 recurrent cases

\begin{tabular}{|c|c|c|c|c|c|c|c|}
\hline Case & Age/sex & Location & Largest diameter & Clinical subtype & $\begin{array}{l}\text { First operative } \\
\text { method }\end{array}$ & $\begin{array}{l}\text { Recurrence } \\
\text { interval (mo) }\end{array}$ & $\begin{array}{l}\text { Second operative } \\
\text { method }\end{array}$ \\
\hline \multicolumn{8}{|l|}{ BCC } \\
\hline & 82/Female & Nasal & 0.5 & Nodular & Primary closure & 97 & FTSG \\
\hline & 76/Female & Nasal & 1.0 & Nodular & FTSG & 128 & FTSG \\
\hline & 45/Male & Oral & 1.5 & Nodular & FTSG & 90 & FTSG \\
\hline \multicolumn{8}{|l|}{ SCC } \\
\hline & 88/Female & Buccal & 2.2 & Well & Local flap & 22 & Local flapa) \\
\hline & 77/Female & Frontal & 1.2 & Unknown & Primary closure & 21 & Primary closure \\
\hline & 78/Male & Zygomatic & 0.5 & Unknown & Primary closure & 15 & Primary closure \\
\hline & 87/Female & Temporal & 1.3 & Poor & Primary closure & 14 & Primary closure \\
\hline & 78/Female & Temporal & 1.0 & Moderate & FTSG & 1 & Lost to follow-upb) \\
\hline & 76/Male & Nasal & 1.5 & Well & FTSG & 1 & Lost to follow-up ${ }^{b)}$ \\
\hline
\end{tabular}

BCC, basal cell carcinoma; FTSG, full thickness skin graft; SCC, squamous cell carcinoma.

a) SCC recurred 10 months after second operation. The patient did not undergo a third operation at our hospital; b) SCC recurred 1 month after first operation. The two patients concerned did not undergo a second operation at our hospital. 
with SCC patients ranging from 54 to 97 years. The mean patient age was 70.8 for BCC and 79.9 years for SCC, respectively. The most popular age group for presenting with BCC was patients in the eighth decade of life (45 patients, 35.2\%), and the peak age for SCC was in the ninth decade (41 patients, $41.8 \%$ ). The male-to-female ratio was 1:1.4 for BCC and 1:2.6 for SCC. In previous studies, the incidence of skin cancer has been shown to be higher for males than females [3,5,7], but recent studies have shown a reversal in this trend. In a retrospective review spanning a time period between 2005 and 2012, Choi et al. [3] found both types of skin cancer to be more common in females, with male-to-female ratios of 1:1.38 for BCC and 1:1.71 for SCC. These greater incidences in women are believed to be due to the greater life expectancy for the female population.

Development of skin cancer may be related to sexual hormones such as estrogen, but such relationship has not been studied extensively in clinical or epidemiologic setting. In an animal study, endogenous estrogen was shown to have a protective effect against skin carcinogenesis [8].

In the present study, the mean tumor diameters was $1.15 \mathrm{~cm}$ for BCC and $1.65 \mathrm{~cm}$ for SCC, and the mean onset-to-diagnosis times was 3.2 and 1.9 months for BCC and SCC, respectively. Typically, SCC is more aggressive in terms of invasion and metastasis than BCC [1], and our results represent the more aggressive characteristics of SCC.

The face is prone to develop skin cancer because it is continuously exposed to UV radiation [9]. In fact, 85\% of BCC and 75\% of SCC occur on the head and neck [1]. Kim et al. [6] reported that the head and neck region accounted for $67.1 \%$ and $21.5 \%$ of all BCC and SCC cases, respectively. In that study, the ratio between BCC and SCC was 3.12:1 for the head and neck region. Choi et al. [10] reported that the head and neck region accounted for $74.5 \%$ and $20.4 \%$ of all BCC and SCC cases, respectively. Our retrospective review had identified total of 148 cases for BCC and 117 cases for SCC. Of these, head and neck region was the anatomic location for 138 (93.2\%) of BCC and 109 (93.2\%) of SCC. Of the 247 cases of all non-melanoma skin cancer in the study, BCC accounted for $55.9 \%$ (138 cases) and SCC for $44.1 \%$ (109 cases). The ratio of BCC to SCC was 1.16:1, which is lower than those reported previously.

Choi et al. [3] retrospectively reviewed 114 cases of BCC and SCC of the face. The authors found that BCC was most common in the nasal unit (37 of 76 cases, $44.1 \%$ ) and that SCC was most common in the buccal unit ( 8 of 38 cases, $21.1 \%$ ). Lee et al. [7] retrospectively reviewed 180 cases of BCC and SCC of the head and neck, and reported that BCC was most common in the nose (63 of 136 cases, $46.3 \%$ ) and that SCC was most common in the cheek and perioral (11 of 44 cases, 25\%, respectively). Our results were comparable to these studies, with $31.9 \%$ of BCC occurring in the nasal unit and $21.1 \%$ of SCC occurring in the buccal unit.

Reconstructions were successful without any significant aesthetic or functional deformity in our patients. Local flaps were most commonly used to cover surgical defects (136/247, 55.1\%), followed by primary closure $(66,26.7 \%)$ and FTSG (38, 15.4\%). In a study involving 137 case of BCC, Lee et al. [11] reported the most frequently used method of reconstruction as local flap (102 cases, 74.4\%), followed by FTSG (25 cases, 18.2\%). In a similar study involving 370 skin cancer cases, the most commonly used method of reconstruction was again local flap (132 cases, 35.7\%) followed by primary closure (63 cases, 17.0\%). Resection wounds too large to close primarily can be covered with full-thickness skin graft, but skin grafts taken from elsewhere usually do not match the color and texture of the adjacent skin. Local flaps can repair relatively large wounds while providing matching skin color and texture. Thus, we had performed $55.1 \%$ of operations using local flaps, with good functional and aesthetic results.

Many factors are associated with recurrence of non-melanoma skin cancers. For BCC, these factors are lesion size $(>10 \mathrm{~mm}$ on a cheek, forehead, scalp, or neck, or of $>6 \mathrm{~mm}$ on the central face, periorbital area, nose, lip, chin, mandible, or auricle), aggressive clinical and histopathologic features (morpheaform, perineural invasion, irregular border), immunosuppressive status, or prior field irradiation $[12,13]$. For squamous cell carcinomas, high risk factors for recurrence are anatomic location (periocular, perioral, ear), lesion diameter $(>2 \mathrm{~cm})$, poor histologic differentiation, rapid growth, and perineural invasion [5,14]. In our review of 247 cases over a 18 -year period, local recurrence had occurred in 9 cases (3.6\%). Disease-specific recurrence rate was $2.2 \%$ (3 cases) for BCC 
and 5.5\% (6 cases) for SCC. These recurrence rates were similar to those from previous skin cancer studies from South Korean population $[10,11,15]$.

In our study, many characteristics of BCC and SCC compared to previously published reports were generally similar, but only different characteristic was the lower ratio of BCC to SCC. Our study have some limitations that small cases and experiences of single institution. Further study can help to establish the characteristics of BCC and SCC, and it will help many surgeons for performing patient consultation and treatment.

\section{REFERENCES}

1. Iorio ML, Ter Louw RP, Kauffman CL, Davison SP. Evidence-based medicine: facial skin malignancy. Plast Reconstr Surg 2013;132:1631-43.

2. Jemal A, Saraiya M, Patel P, Cherala SS, Barnholtz-Sloan J, Kim J, et al. Recent trends in cutaneous melanoma incidence and death rates in the United States, 1992-2006. J Am Acad Dermatol 2011;65:S17-25.el-3.

3. Choi JH, Kim YJ, Kim H, Nam SH, Choi YW. Distribution of Basal cell carcinoma and squamous cell carcinoma by facial esthetic unit. Arch Plast Surg 2013;40:387-91.

4. Netscher DT, Spira M. Basal cell carcinoma: an overview of tumor biology and treatment. Plast Reconstr Surg 2004;113:74e-94e.

5. Rudolph R, Zelac DE. Squamous cell carcinoma of the skin. Plast Reconstr Surg 2004;114:82e-94e.
6. Kim HS, Cho EA, Bae JM, Yu DS, Oh ST, Kang H, et al. Recent trend in the incidence of premalignant and malignant skin lesions in Korea between 1991 and 2006. J Korean Med Sci 2010;25:924-9.

7. Lee TS, Pyon JK, Mun GH, Bang SI, Oh KS, Lim SY. A retrospective clinical study of skin cancer: a single institution's experience on 370 cases. J Korean Soc Plast Reconstr Surg 2008;35:261-6.

8. Mancuso M, Gallo D, Leonardi S, Pierdomenico M, Pasquali E, De Stefano I, et al. Modulation of basal and squamous cell carcinoma by endogenous estrogen in mouse models of skin cancer. Carcinogenesis 2009;30:340-7.

9. Jin HR, Lee JY, Lee DW, Shin SO, Choi YS, Yoo SJ, et al. Primary facial skin cancer: clinical characteristics and surgical outcome in Chungbuk Province, Korea. J Korean Med Sci 2005;20:279-82.

10. Choi KR, Lee JH, Kim DY, Lee SY, Cho BH. Clinical study of facial skin cancers. J Korean Soc Plast Reconstr Surg 1997;24:734-40.

11. Lee BM, Shim JS, Kim TS, Han DG, Park DH. Clinical consideration of 137 cases of basal cell carcinoma in face. Arch Craniofac Surg 2013;14:107-10.

12. National Comprehensive Cancer Network Basal Cell and Squamous Cell Skin Cancers. Basal cell and squamous cell skin cancers: clinical practice guidelines in oncology. J Natl Compr Canc Netw 2004;2:6-27.

13. Silverman MK, Kopf AW, Bart RS, Grin CM, Levenstein MS. Recurrence rates of treated basal cell carcinomas. Part 3: Surgical excision. J Dermatol Surg Oncol 1992;18:471-6.

14. Brodland DG, Zitelli JA. Surgical margins for excision of primary cutaneous squamous cell carcinoma. J Am Acad Dermatol 1992;27:241-8.

15. Lee TS, Pyon JK, Mun GH, Bang SI, Oh KS, Lim SY. A clinical review on 143 cases of basal cell carcinoma. J Korean Soc Plast Reconstr Surg 2008;35:698-702. 